\title{
A Literature Review on Supramolecular Devices
}

\author{
Siu-Kan Law ${ }^{1(\mathbb{D})}$, Ka-Ho Hui ${ }^{1(\mathbb{D})}$, Yat-Hei Fung ${ }^{1(\mathbb{D})}$, Wai-Tung Leung ${ }^{1(\mathbb{D})}$, Jie Han ${ }^{1, * \mathbb{C}}$, \\ Chui-Man Lo ${ }^{1,2, *}$ (D) \\ 1 Department of Science, School of Science and Technology, Hong Kong Metropolitan University, Ho Man Tin, Kowloon, \\ Hong Kong, China \\ 2 Department of Chemistry, The Chinese University of Hong Kong, Shatin, New Territories, Hong Kong, China \\ * Correspondence: cmlo@cuhk.edu.hk (C.-M.L.); chan@hkmu.edu.hk (J.H.);
}

Scopus Author ID 57199890667 (J.H.)

57193752045 (C.-M.L.)

Received: 22.09.2021; Revised: 20.10.2021; Accepted: 24.10.2021; Published: 20.11.2021

\begin{abstract}
Supramolecular device includes hydrogel and polymer manufactured by gelation or polymerization. Hydrogels are produced via chemical and physical gelation. Polymers are synthesized through photopolymerization, step-growth, and chain-growth polymerization. The supramolecular device possesses as the host to entrap the guest molecules via chemical, physical and physical-chemical microencapsulation. The present review article discusses the microencapsulation techniques, mechanism of gelation or polymerization, and different gelation or polymerization methods with given examples, such as gelatin, chitosan, and polyvinyl alcohol (PVA), and polyamide to act as the hosts for encapsulation.
\end{abstract}

Keywords: microencapsulation; polymerization; gelation; supramolecular devices; physical crosslinking; chemical cross-linking.

(C) 2021 by the authors. This article is an open-access article distributed under the terms and conditions of the Creative Commons Attribution (CC BY) license (https://creativecommons.org/licenses/by/4.0/).

\section{Introduction}

Supramolecular devices are used in material applications beyond the limitations of conventional polymers. Its monomer units consist of conventional and non-conventional polymers connected by reversible and highly directed secondary interactions. Convention polymers are linked by strong covalent bonds between the monomer units and have excellent material properties, while non-conventional polymers are formed by non-covalent interaction, non-covalent interactions such as hydrogen bonding, metal coordination, and $\pi-\pi$ (or arenearene) interactions $[1,2]$.

However, entanglement polymers with high-viscosity melts, high temperatures, and high pressures are usually required for processing. Supramolecular polymers combine good material properties with a low viscosity melt which is easy to handle. They have unique characteristics such as self-repair fractures. Covalent polymers are recycled materials because of their strong covalent bonds, and it is never broken down [3-5].

Hydrogel-based devices are used as a carrier to prolong their functions. Hydrogel is a three-dimensional hydrophilic polymer network made up of water-soluble polymers, crosslinked to form a water-insoluble hydrogel (Fig. 1). The characteristics of hydrogels are swelling and retaining a portion of water when placed in an aqueous solution at least $20 \%$ of water and reach values of $99 \%$ by weight, termed as superabsorbent. Hydrogels are produced by physical or chemical cross-linking polymers. Its cross-link is formed by covalent or non-covalent 
interactions. Covalently cross-linked hydrogels are also called 'permanent or 'chemical' gels [6-8].

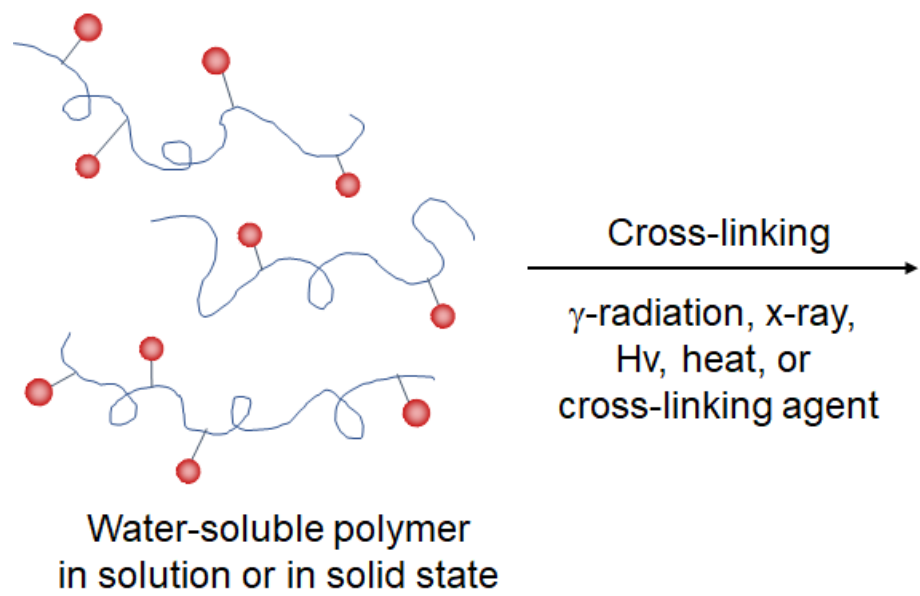

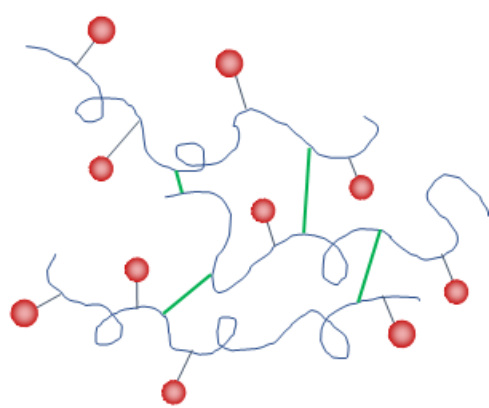

Hydrogel

Figure 1. Synthesis of hydrogels by cross-linking of water-soluble polymers.

\section{Polymers}

There are two types of polymers, including natural and synthetic. Natural Polymeric materials such as DNA, protein, and wood are biopolymers produced by a living organism. Biopolymers contain monomeric units which are covalently bonded to form larger structures, e.g., amylose. It is a polysaccharide made of $\alpha$-D-glucose units by a covalent bond (Fig. 2) $[9,10]$.<smiles>CC(C)CC(=O)O</smiles><smiles>CCOC(CC)CC(=O)O</smiles>

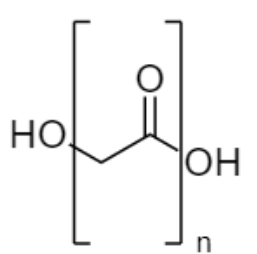<smiles>CCOC1OC(CO)C(O)C(O)C1O</smiles><smiles>CCC1OC(OC2C(CO)OC(CO)C(O)C2O)C(O)C(O)C1O</smiles>

Figure 2. Chemical structure of some biopolymers.

\subsection{Gelation.}

Refer to the linkage of macromolecular chains, which depends on the structure and conformation of starting material, eventually leading to the gradual formation of larger branched polymers (Fig. 3) [11]. Branched polymers are formed linkage between the chains to form larger polymers. As the connection continues, a larger branched polymer is produced. Macromolecules are formed under a certain degree of reactive connection between the polymers. At the reaction point (defined as the gel point), the system loses fluidity, and its viscosity becomes very large [12-14]. 




Figure 3. Polymers after cross-linking.

The mixture of polydisperse soluble branched polymer is referred to as 'sol'. Continuation of the linking process increases the size of the branched polymer; it causes decreasing solubility of polymers. This 'infinite polymer' acts as 'gel' or 'network', a fully branched polymer. Sol-gel transition or gelation is a process that transit the branched polymer to infinite molecules. The gel point is a critical point where it first appears [15].

\subsection{Physical gelation.}

The formation of gel from the branched polymer by physical linking is a physical linking formed by non-covalent interactions. Fig. 4 is shown three types of physical cross-link, including hydrophobic interactions, charge interactions, and hydrogen bonds within the hydrogel-based devices. Hydrophobic interactions are the hydrophobic blocks aggregate to drive in physical gelation when the temperature increases (Fig. 4a). Charge interaction involves adding polymer with opposite charge or small molecule cross-linker of opposite charge to drive in physical gelation (Fig. 4b). Physical gelation is interacted by forming hydrogen bonds between polymers (Fig. 4c.) [6].

(a)

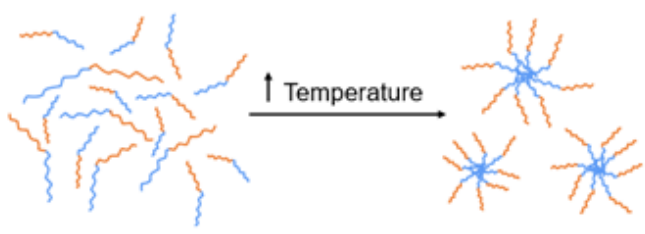

(b)



(c)

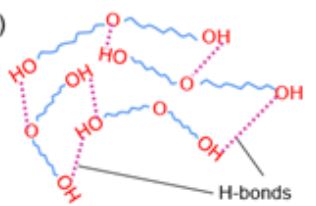

Figure 4. Physical cross-link.

\subsection{Chemical gelation.}

The formation of gel from the branched polymer is chemical linking which involves the formation of covalent bonds to form a strong gel. Most chemical hydrogels are produced by condensation and vulcanization.

Branched polymers with hydrated surfaces form chain networks through condensation reactions. Water molecules exist in these reactions. Long and viscoelasticity molecules are not connected, allowing relative movement between them. The vulcanization process is triggered by heat. Molecules of the elastomer break its double bonds and form new covalent or ionic bonds with other molecules to have a three-dimensional network during vulcanization (Fig. 5) $[16,17]$. 


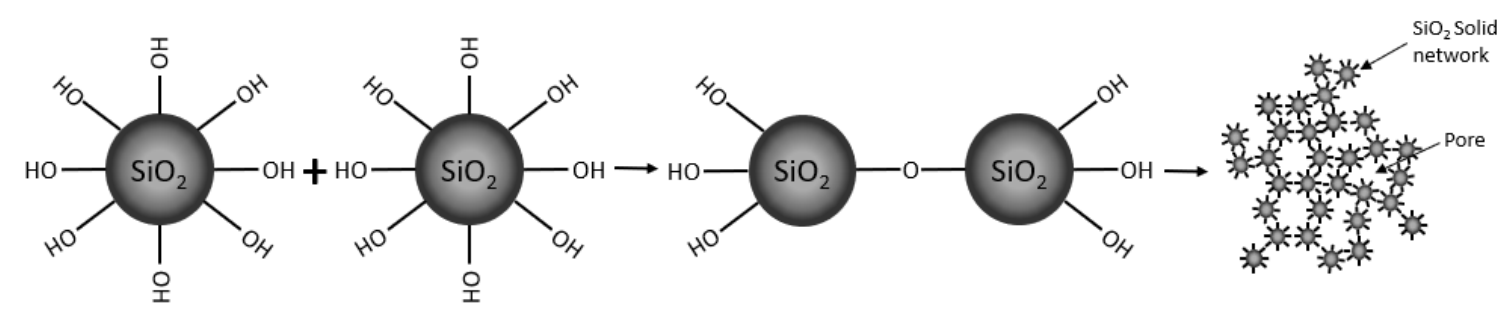

Figure 5. Condensation and gelation model.

\section{Swellability of Hydrogels}

The hydrophilic nature exists in branched polymers, such as gelatin, chitosan, and polyvinyl acetate, because of hydrophilic functional groups, including amino, carboxyl, and hydroxyl groups. When the hydrogel comes in contact with water, it diffuses into the polymer hydrogel and causes the hydrogel to swell [18].

Hydrogel possesses good swelling behavior as the temperature gets high. As the temperature increases, the segment mobility of the gelatin chain increase leading to the formation of more voids in the composite hydrogel. More water molecules penetrate the composite hydrogel, resulting in a higher swelling capacity [19].

Hydrogels show high swelling properties at low $\mathrm{pH}$ if hydrogel possesses the amino group. At low $\mathrm{pH}$ values, the amino groups protonate, causing repulsive forces in the polymer chain. The dissociation of secondary interactions such as intramolecular hydrogen bonds allows more water to enter into the gel. At high $\mathrm{pH}$ values, amino groups eliminate the repulsive forces between polymer chains. It leads to shrinkage of the polymer matrix, causing limitation of water intake [20].

The Flory-Rehner equation is equilibrium swelling theory for describing the mixing of polymers and liquid molecules. The equilibrium stage of the hydrogel swollen in a fluid is determined by two reverse forces, e.g., the thermodynamic force of mixing favors swelling; another force is the stored force in the stretched polymer chains blocking swelling (Eq. 1) [21].

$$
\Delta \mathrm{G}_{\text {total }}=\Delta \mathrm{G}_{\text {elastic }}+\Delta \mathrm{G}_{\text {mixing }}
$$

\subsection{Stability of hydrogels.}

Hydrogel with different cross-linkers is more stable. Multi-crosslinker form multiple covalent linkages, such as ester linkages. It produces a stable three-dimensional hydrogel network. Cross-linking introduces different functional groups into the hydrogel matrix to increase the number of active sites on the hydrogel. Thermal energy is not sufficient to break the covalent linkages resulting from the reaction between the branched polymers and crosslinkers. Hydrogels with physical cross-links are weaker than with cross-linkers. Physical crosslink hydrogel only exists intramolecular force, which is weaker than a covalent bond. Thermal energy may be sufficient to break the intramolecular force. Thus, the structure of hydrogel with cross-linker is more stable than with physical cross-link [22,23].

\subsection{Gelatin hydrogels.}

Gelatin (Fig. 6) is a heterogeneous mixture of single-chain or multi-chain polypeptides. It has $\mathrm{NH}_{2}$ amino group with a 7-21 nm pore size [24,25]. 


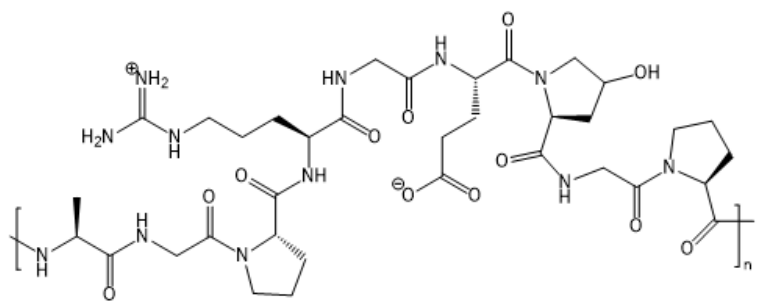

Figure 6. Chemical structure of gelatin.

This is also a mixture of peptides and proteins. These peptides and proteins are produced by the partial hydrolysis of collagen extracted from animals' skin, bones, and connective tissues, e.g., cows, chickens, pigs, and fish. The natural molecular bonds between individual collagen chains are broken down into a form and are easier to rearrange during hydrolysis. Gelatin is almost odorless and tasteless, and its appearance is colorless or light yellow. It is transparent and brittle and appears in flake or powder form. Polar solvents (hot water, glycerin, and acetic acid) may dissolve gelatin but are insoluble in organic solvents such as alcohol.

Gelatin is extracted by acidic and alkaline. In acidic extraction, rinsing the fish skin in running tap water for 1 hour to remove excess material. The skin/solution ratio is 1:7, and the cleaned raw materials are soaked in low-mole $\mathrm{NaOH}(0.4 \mathrm{~N})$ at room temperature for 4 hours. This solution is neutralized after a given treatment time. The treated material is mixed with distilled water in a ratio of $1: 2$ and extracted at $70^{\circ} \mathrm{C}$ for 1.5 hours, then filtered by using a double-layer cheese filter cloth. The filtrate is dried in a hot air oven at $50^{\circ} \mathrm{C}$.

In alkaline extraction, the fish skin is washed in running water, dried, and immersed in a saturated $\mathrm{Ca}(\mathrm{OH})_{2}$ solution at a ratio of $1: 2$ at $20^{\circ} \mathrm{C}$ for 14 days. By maintaining the $\mathrm{pH}$ of $\sim 10$ washing materials, too much lime is removed. The skin is soaked in distilled water overnight for dissolving the gelatin at $48^{\circ} \mathrm{C}$. This material is filtered using filter paper. The filtrate is passed through a cation exchange resin again to lower the $\mathrm{pH} \sim 5$ then freeze-dried [26].

\subsubsection{Mechanism of gelatin gel preparation.}

Gelatin gel is prepared by physical and chemical gelation. In physical gelation, branched gelatin polymers exist in water at $50^{\circ} \mathrm{C}$. Physical cross-linking such as ionic and hydrogen bonding interactions between gelatin molecules are reinforced during cooling. After cooling, branched gelatin molecules formed triple helices. Physical cross-linking is continuously reinforced as the temperature gets lower. At the last, branched gelatin molecules formed a gel network (Fig. 7) [27].

In chemical gelation, gelatin and glutaraldehyde act as monomers and cross-linkers, respectively. The cross-linking of gelatin and glutaraldehyde occurs at the amino group of gelatin. Gelatin acts as a nucleophile, and the individual electron pairs of the nitrogen atom attack the carbonyl carbon of glutaraldehyde. In step $2, \mathrm{O}^{-}$receive the proton from $\mathrm{NH}^{2+}$ to form amino aldehyde. $\mathrm{NH}^{2+}$ reduce to secondary amide $\left(2^{\circ}\right.$ amide $)$ and hydronium ion $\left(\mathrm{H}_{3} \mathrm{O}^{+}\right)$. In step 3, the hydroxyl group next to the secondary amine group receives proton from $\mathrm{H}_{3} \mathrm{O}^{+}$. $\mathrm{OH}^{-}$become to $\mathrm{H}_{2} \mathrm{O}^{+} .2^{\circ}$ amide group donate a lone pair electron to form $\mathrm{N}=\mathrm{C}$. The bonding between carbon and oxygen is broken. In step 4, the water molecule is produced, and gelatin hydrogel is formed [28]. 


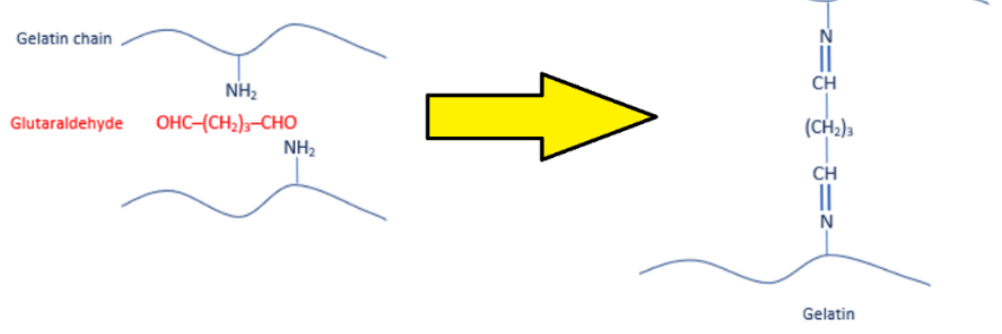

Figure 7. Chemical cross-link of gelatin with glutaraldehyde.

\subsection{Chitosan hydrogels.}

Chitosan is produced by a glycosidic backbone. It has an $\mathrm{NH}_{2}$ amino group, and the repeating units of chitosan are glucosamine and $\mathrm{N}$-acetylated glucosamine. Its pore sizes are $150 \mu \mathrm{m}$ (Fig. 8).

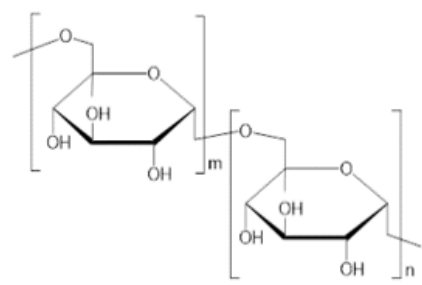

Figure 8. Chemical structure of chitosan.

Chitin is usually extracted from the shells of crustaceans. The crushed material is treated with acid to dissociate the calcium carbonate structure completely. It is then subjected to alkaline extraction for achieving protein solubilization. In the subsequent purification step, it obtained from the deproteinization process undergoes a decolorization step to remove pigment residues and obtain a colorless product [29].

Chitosan is a water-insoluble polymer and a deacetylated derivative of chitin found from insect exoskeletons, outer shells of crabs, and fungal cell walls. It is non-toxic, odorless, and biocompatible in animal tissues and applies in the medical and pharmaceutical fields. This is slightly bound with the water into chitosan $[30,31]$.

\subsubsection{Mechanism of chitosan hydrogel preparation.}

Chitosan and glutaraldehyde act as monomers and cross-linkers, respectively. The cross-linking of chitosan and glutaraldehyde occurs at the amino group of chitosan. Chitosan acts as a nucleophile, and the individual electron pairs of the nitrogen atom attack the carbonyl carbon of glutaraldehyde. Step 2, $\mathrm{O}^{-}$receives the proton from $\mathrm{NH}_{2}{ }^{+}$to form amino aldehyde. Also, $\mathrm{NH}_{2}{ }^{+}$reduce to secondary amide $\left(2^{\circ}\right.$ amide) and hydronium ion $\left(\mathrm{H}_{3} \mathrm{O}^{+}\right)$. Step 3 , the hydroxyl group next to the secondary amine group receives proton from $\mathrm{H}_{3} \mathrm{O}^{+}$. $\mathrm{OH}^{-}$become $\mathrm{H}_{2} \mathrm{O}^{+} .2^{\circ}$ amide group donate a lone pair electron to form $\mathrm{N}=\mathrm{C}$. The bonding between carbon and oxygen is broken. Step 4, the water molecule is produced, and chitosan hydrogel is formed (Fig. 9) [21,32]. 


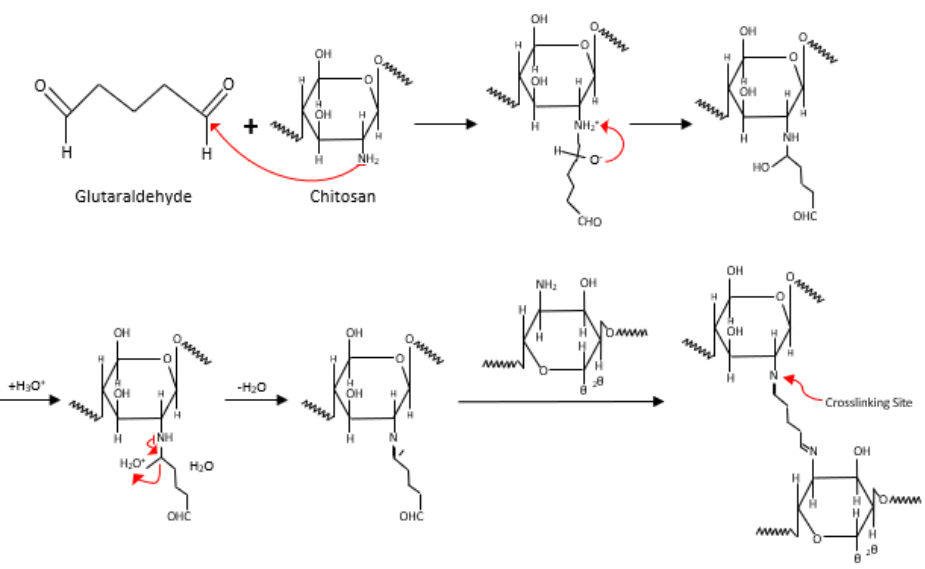

Figure 9. Reaction mechanism between the amino group of chitosan and carbonyl groups of glutaraldehyde.

\subsection{Polyvinyl acetate hydrogels.}

Polyvinyl acetate (PVA) is a water-soluble synthetic polymer, which possesses a hydroxyl group (Fig. 10) [33].

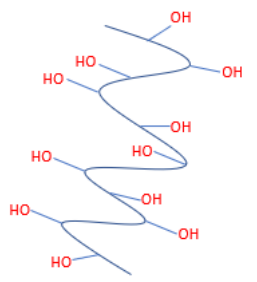

Figure 10. Chemical structure of PVA.

It is used for papermaking, textile warp sizing, and various coatings. Colorless, tasteless, and usually provided in the form of beads or an aqueous solution [34]. PVA has biocompatibility, low protein adhesion tendency, and low toxicity, so it is used in various medical applications, including cartilage substitutes, contact lenses, and eye drops [35].

PVOH cannot be prepared by polymerizing vinyl alcohol because the substance does not exist in a free state. However, it is synthesized by partially or completely hydrolyzing polyvinyl acetate to remove acetate groups [34]. Ethanol act as a nucleophile, lone pair electron of hydroxylic oxygen attack carboxylic carbon. The double bond between carbon and oxygen is broken. Bond electron is withdrawing to carboxylic oxygen and reduces to $\mathrm{O}^{-}$. It donates lone pair electron to carbon atom causes breaking $\mathrm{C}-\mathrm{O}$ bond and PVA is produced via hydrolysis (Fig. 11) [36].

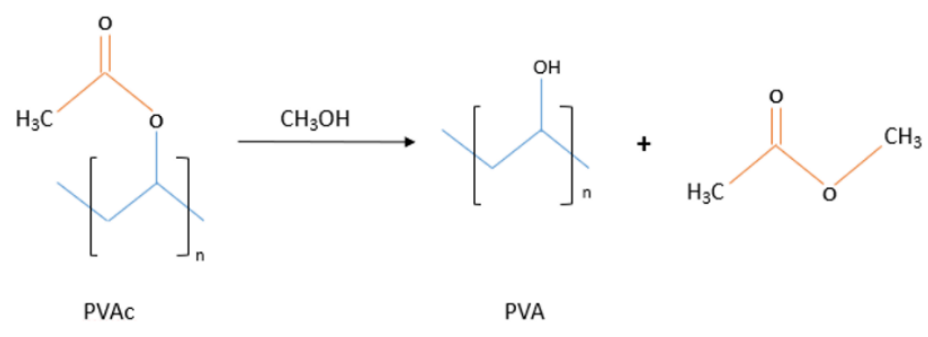

Figure 11. Hydrolysis of PVAc with methanol produces PVA.

\subsubsection{Mechanism of polyvinyl acetate hydrogel preparation.}

Polyvinyl acetate, organic acid, and sulfonic acid act as monomers, cross-linkers, and catalysts, respectively. The cross-linking of PVA and organic acid occurs at the hydroxyl group of PVA. Step 1 is the formation of a cation. The double bond from $\mathrm{C}=\mathrm{O}$ donates a lone pair 
electron to proton from strong acid such as sulfonic acid. Step 2 occurs the delocalized carbocation. Carboxyl oxygen from organic acid gets protonated to give delocalized carbocation making the carbocation a better electrophile. PVA acts as a nucleophile, and the lone electron pairs of the hydroxyl oxygen attack the carbocation of organic acids. Step 3 is the protonation. A proton of the hydroxyl group from PVA is transferred to one of the hydroxyl groups from organic acid to form a good leaving group. Step 4 is the formation of a $\pi$ bond. Hydroxy oxygen donates a pair of electrons to a carbon atom for making a $\pi$ bond by eliminating water. Step 5 is the ester formation, $\mathrm{O}^{+}-\mathrm{H}$ bond is broken and donated to $\mathrm{O}^{+}$through receiving proton by a conjugated base (Fig. 12) [37].
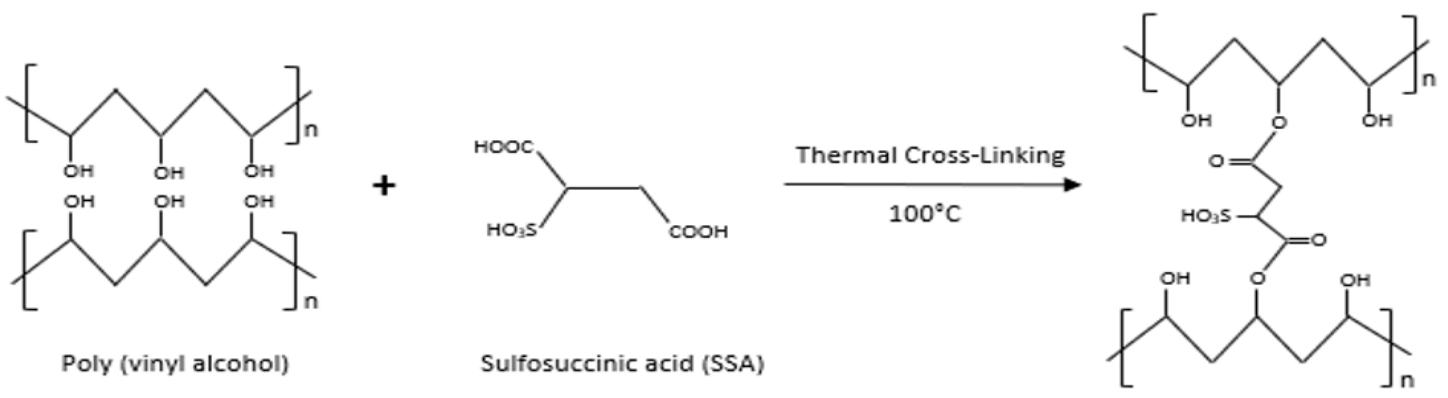

Figure 12. Mechanism of PVA/organic acid reaction.

\section{Polymer-Based Device}

Polymer is a class of macromolecules with high molecular weight and has a wide range of properties. Its synthetic and natural polymers play a vital role in daily life. They consist of multiple repeating units of low molecular weight monomers. The macromolecular mass produces unique physical properties, including toughness, high elasticity, viscoelasticity. It tends to form amorphous and semi-crystalline structures rather than crystals.

Synthetic polymers such as polyethylene, polypropylene, and polystyrene are commonly used in clothing and plastics. The backbone of these synthetic polymers is made up of carbon-carbon bonds through polymerization (Fig. 13) [38].

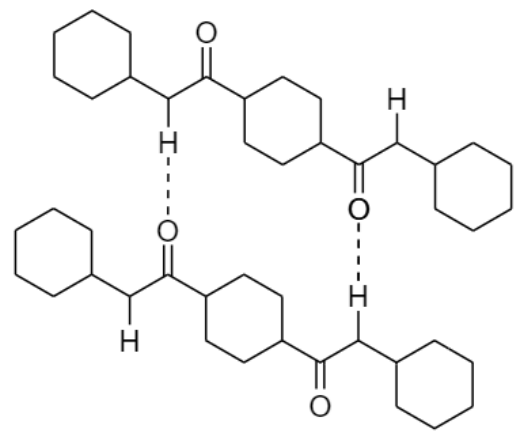

Figure 13. Chemical structure of synthetic polymers.

Various polymer structures are produced depending on the physical arrangement of monomers. The physical arrangement of monomer contains five types: linear macromolecule, branched macromolecule, the semi-crystalline structure of polymer, elastomer, and thermoset, within the polymer backbone. A linear polymer is simply a macromolecular chain where all monomers exist in a single line with no branches or intramolecular bridges [39]. Branched polymers are defined as having secondary polymer chains linked to a primary backbone, resulting in a variety of polymer architectures such as star, H-shaped, pom-pom, and combshaped polymers [40]. Semi-crystalline polymers are defined that the material exhibits 
organized and tightly packed molecular chains. Elastomer is defined as a cross-linked and amorphous polymer that only has very weak intermolecular forces within the polymer backbone (Fig. 14) [41]. Thermosetting polymers are irreversibly hardened by curing a soft solid or viscous liquid prepolymer or resin via heat, suitable radiation, and catalyst [42].



Liner macromolecule

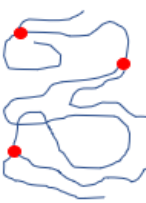

Branched

macromolecule

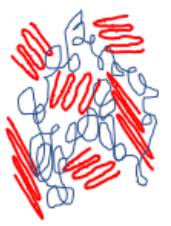

Semi-crystalline

polymer

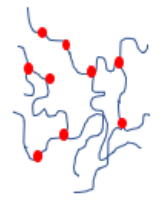

Elastomer

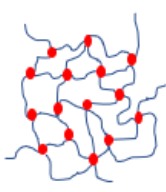

Thermoset

Figure 14. Microstructures of polymers.

\subsection{Polymerization.}

Polymerization is a process of bonding monomer molecules together by covalent bonds to form polymer chains or three-dimensional networks [43]. Polymerization divides into two classes, which are step-growth, chain growth, and photopolymerization.

\subsubsection{Step-growth polymerization.}

Step-growth polymerization refers to a polymerization mechanism in which monomers react with monomers to form first dimers; dimers react with monomer to form trimers; trimers react with monomer to form a tetramer on, then oligomers are formed. The polymer backbone is formed (Fig. 15). Many are naturally occurring, and some synthetic polymers are produced by step-growth polymerization, such as polyesters, polyamides, and polyurethane [44].

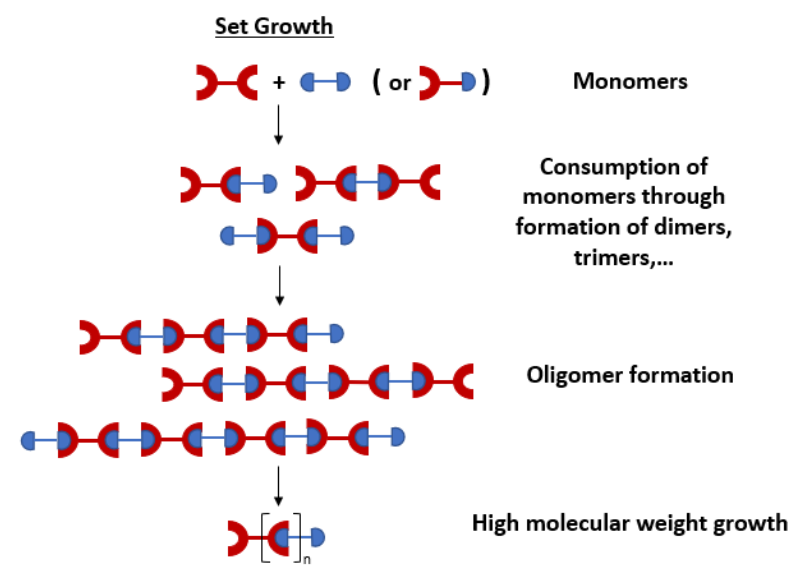

Figure 15. Step-growth polymerization.

\subsubsection{Chain-growth polymerization.}

Chain-growth polymerization is divided into four steps: chain initiation, chain propagation, chain transfer, and chain termination. In Fig., chain initiation is the process of initially generating chain carriers in chain polymerization (chain carriers are certain intermediates in the chain propagation process, such as free radicals and ions); chain propagation acts as the active center on the growing polymer molecule, adding a monomer molecule to form a new polymer molecule; chain transfer is the active center of polymer A takes one atom from molecule $\mathrm{B}$ and terminates in chain polymerization; chain termination is 
that active center disappears in the chain polymerization process, which causes termination of chain propagation (Fig. 16) [45].

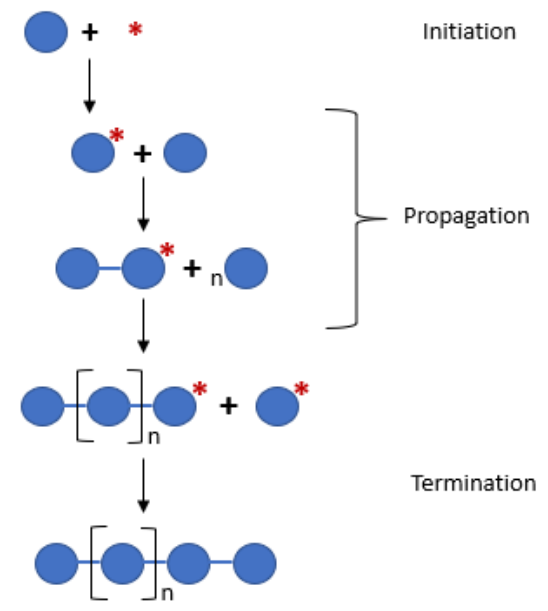

Figure 16. Chain-growth polymerization.

\subsection{Photopolymerization.}

Photopolymer is a polymer that usually changes its properties when exposed to light in the ultraviolet or visible region of the electromagnetic spectrum. An interaction has occurred between monomers, oligomers, and photoinitiators during photopolymerization. The monomer reacts with other monomer molecules to form a larger polymer chain or three-dimensional network. Oligomers composed of several similar or identical repeating units are actually or conceptually derived from replicas of their monomers. Photoinitiator generates reactive species, such as free radicals, cations, or anions, when exposed to radiation like ultraviolet or visible light. Photopolymerization divides into ionic and free radicals.

In ionic photopolymerization, the proposed cationic photopolymerization mechanism starts with the light excitation of the initiator. Once excited, homogeneous cleavage and dissociation of the counter anion occur, generating cationic radicals $(\mathrm{R})$, aryl groups ( $\left.\mathrm{R}^{\prime}\right)$, and unaltered counter anions $(\mathrm{X})$. The extraction of Lewis acid by cationic radicals produces very weakly bound hydrogen and radicals. The acid is further deprotonated by the anion (X) in the solution to generate a Lewis acid with the starting anion $(\mathrm{X})$ as the counterion. It is believed that the acidic protons produced eventually initiate polymerization (Eq. 2) [46].

hv

$\mathrm{MH}$

$$
\left[\mathrm{R}^{\prime}-\mathrm{R}^{+} \mathrm{X}^{-}\right] \rightarrow\left[\mathrm{R}^{\prime}-\mathrm{R}^{+} \mathrm{X}^{-}\right] \rightarrow \mathrm{R}^{+}+\mathrm{R}^{\prime}+\mathrm{X}^{-} \rightarrow \mathrm{R}^{+}-\mathrm{H}+\mathrm{R}^{\prime}-\mathrm{M}+\mathrm{X}^{-} \rightarrow \mathrm{R}+\mathrm{H}^{+} \mathrm{X}^{-}
$$

In free radical polymerization, the light is absorbed by the photoinitiator result in the generation of free radicals, which cause the cross-linking reaction of the mixture of functionalized oligomers and monomers, thereby forming a cured film. The radical mechanism for chain growth polymerization includes three basic steps: initiation, chain growth, and chain termination [45].

\subsubsection{Polyamide.}

Polyamides are polymers with repeating units connected by amide bonds. Polyamides exist naturally and artificially. Examples of natural polyamides are proteins, such as wool and silk. Artificial polyamides can be made by stepwise polymerization or solid-phase syntheses, 
such as nylon, aramid, and sodium poly-aspartate. Synthetic polyamides are often used in textiles, the automotive industry, carpets, kitchen utensils, and sportswear due to their high durability and strength. They can be aliphatic, semi-aromatic, or fully aromatic thermoplastics. Compared with other aliphatic amides (nylons), aramid has higher strength, better solvent resistance, flame resistance, heat resistance, and greater dimensional stability than other aliphatic amides (nylons). However, it is expensive and difficult to produce (Fig. 17) [47].

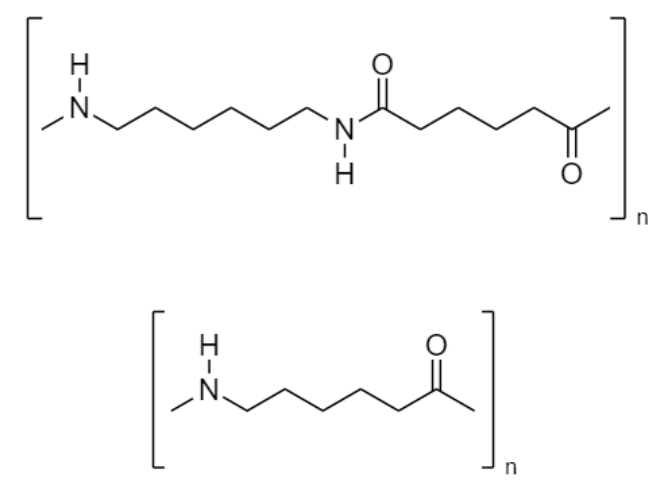

Figure 17. Chemical structure of polyamides.

Polyamides are prepared by condensation reaction. Two amino acids are combined in a condensation reaction. A covalent bond is formed between the amine nitrogen of one amino acid and the carboxyl carbon of the second amino acid. The water molecules are then removed as the second product. This condensation reaction is continuously replied to, and a polyamide is formed finally (Fig. 18) [48].

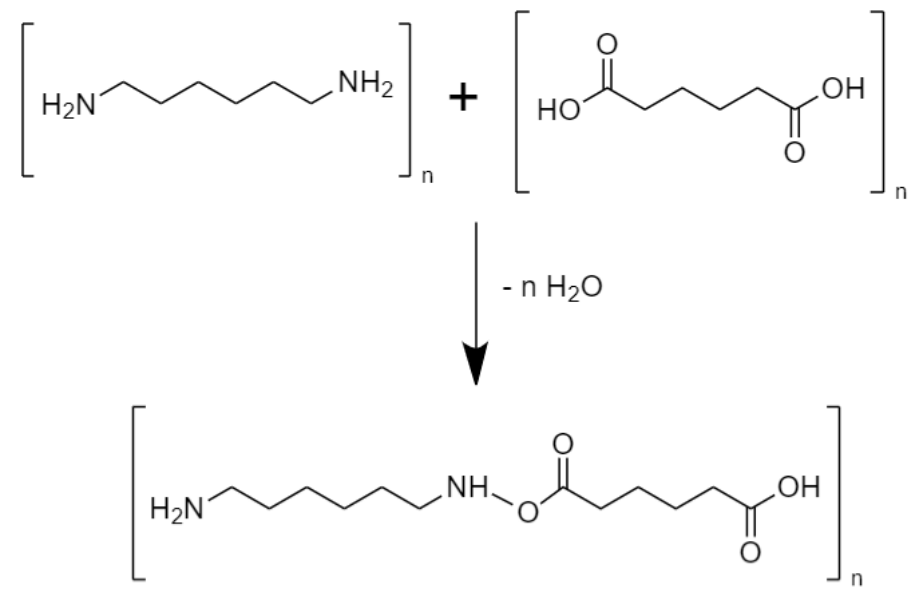

Figure 18. Chemical formula of polyamide.

\subsubsection{Mechanism of polyamide preparation.}

Ethylenediamine and sebacoyl chloride act as monomers and cross-linkers, respectively, and the cross-linking occurs at the amino group of ethylenediamine. Ethylenediamine acts as a nucleophile, and the individual electron pairs of the nitrogen atom attack the carbonyl carbon of sebacoyl chloride. Carbonyl oxygen becomes $\mathrm{O}^{-}$and carbonyl carbon acts as carbocation. Step 2, $\mathrm{O}^{-}$donates lone pair electron to carbon atom form $\mathrm{C}=\mathrm{O}$ and $\mathrm{Cl}$ as a good leaving group become free $\mathrm{Cl}^{-}$. Meanwhile, free $\mathrm{Cl}^{-}$receives the proton from an amino group. $\mathrm{N}-\mathrm{H}$ bonding is broken, and the bone electron donates to the carbocation. It reduces to a carbon atom. Step 3, polyamide is formed, and hydrochloric acid is produced. The 
cross-link between ethylenediamide and sebacoyl chloride continuously occurs to form polyamide (Fig. 19) [49].

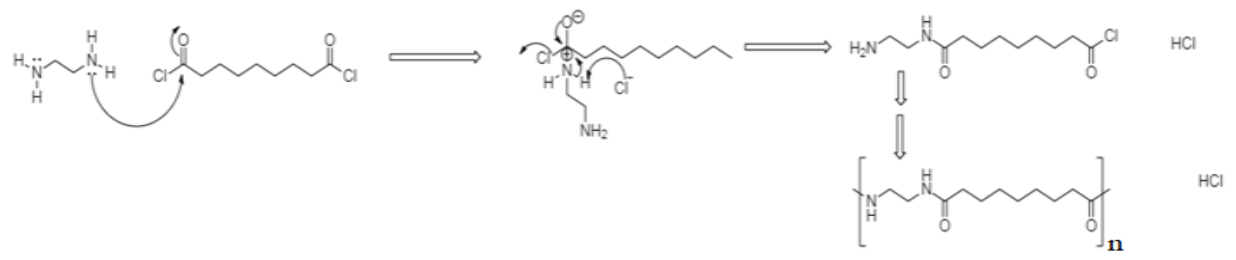

Figure 19. Cross-linking between ethylenediamine and sebacoyl chloride.

\subsection{Chemical method.}

This is divided into interfacial polymerization and in situ polymerization. In interfacial polymerization, a solution containing the active compound and water-soluble monomer A with distilled water is prepared. An oil-in-water (W/O) emulsion is formed by emulsifying the water phase in the organic outer phase. The organic soluble monomer B is added to the organic phase, and an interfacial polycondensation reaction between the two monomers starts at the O/W interface (Fig. 20) [50].

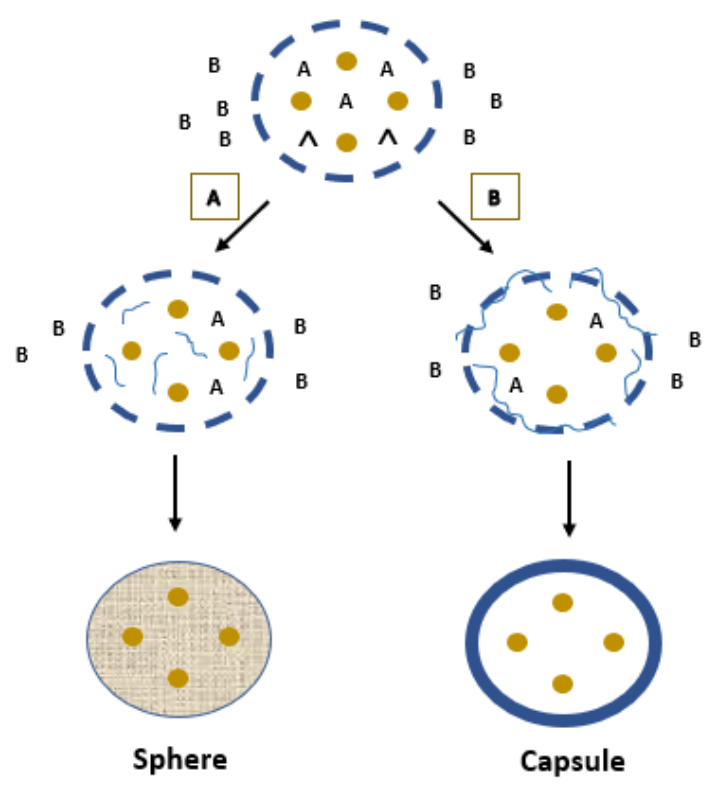

Figure 20. Principle of the microencapsulation by interfacial polymerization.

In situ polymerization, the prepolymer solution was poured into the emulsion system, the prepolymer and hydration dispersed in the continuous aqueous phase cause migration of polymers via hydrogen bonding. Then, the positively charged prepolymer was formed. The oil droplets were surrounded evenly by macromolecules. At the early stage, cross-linking of prepolymers occurs via electric attraction between prepolymers. An electric attraction between prepolymers becomes weaker after a certain reaction period; a small amount of prepolymer would form nanoparticles. Nanoparticles deposit on the shell surface via self-polymerization of the surface and a core-shell configuration is formed (Fig. 21) [51].

\subsection{Physical method.}

This divides into spray drying and congealing, fluidized-bed technology, and solvent evaporation. Spray drying and congealing is the core particles dispersed in a polymer solution 
and sprayed into a hot chamber. The shell material solidifies onto the core particles as the solvent evaporates.

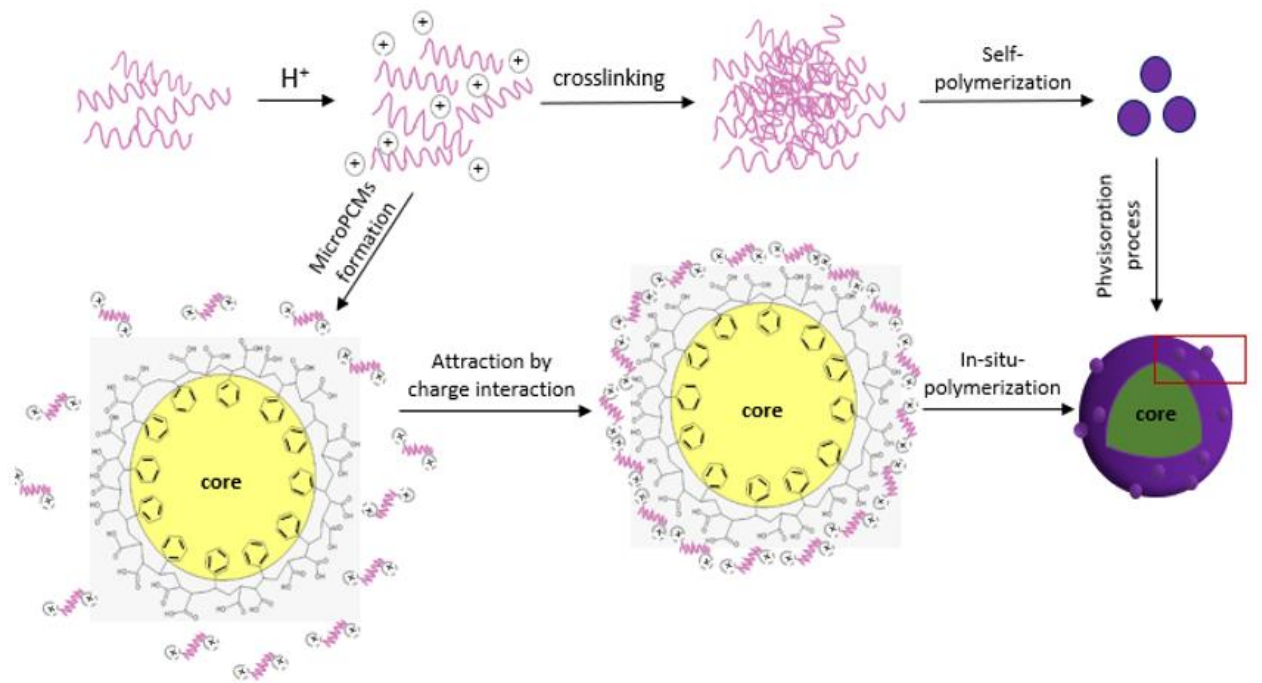

Figure 21. Diagram of microencapsulation by in situ polymerization.

Coating solidification in spray drying is affected by the quick evaporation of a solvent in which the coating material is dissolved. Coating solidification in the spray method is completed by thermally congealing the molten coating material or introducing the coating core material mixture into a non-solvent to solidify a dissolved coating. The microcapsules are obtained (Fig. 22).

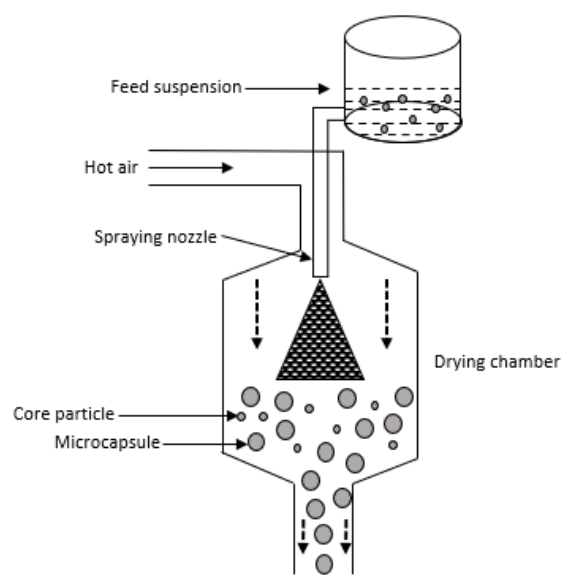

Figure 22. Process of microencapsulation by spray-drying.

Fluidized-bed technology is the liquid coating sprayed onto the particles, and the rapid evaporation helps form an outer layer on the particles. This thickness and formula of the coating were obtained as required. Different fluidized bed coaters include top spray, bottom spray, and tangential spray (Fig. 23).

Top spray paints down onto the fluidized bed when the solid or porous particles move to the paint area then encapsulated. Bottom spray particles move upward through a perforated bottom plate, and the nozzle area sprays on the coating material, which is wrapped by the coating material. Tangential spray particles move through the gap created by raising the disc into the spraying zone and are encapsulated [52]. 

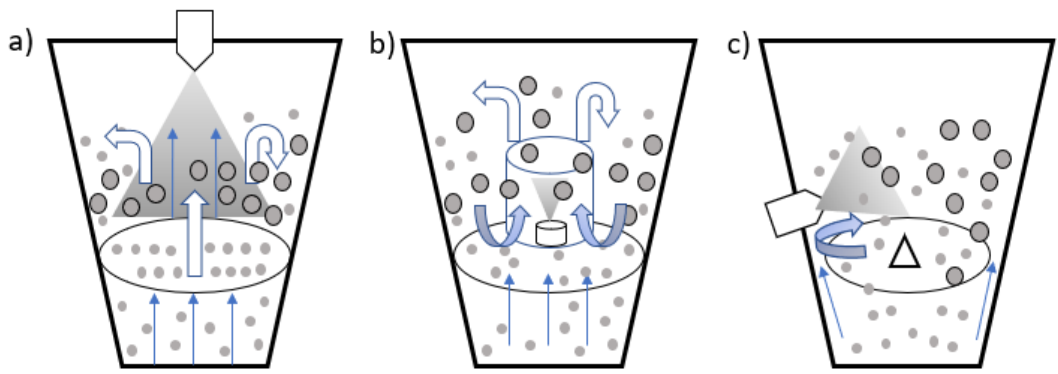

Figure 23. Schematics of a fluid-bed coater. (a) Top spray; (b) bottom spray; (c) tangential spray.

Solvent evaporation is the core material dissolved or dispersed in the coating polymer solution. This mixture is added to the liquid manufacturing vehicle phase with stirring; the mixture is heated to evaporate the solvent for the polymer. The coated material shrinks surrounding the core material and is encapsulated (Fig. 24) [53].

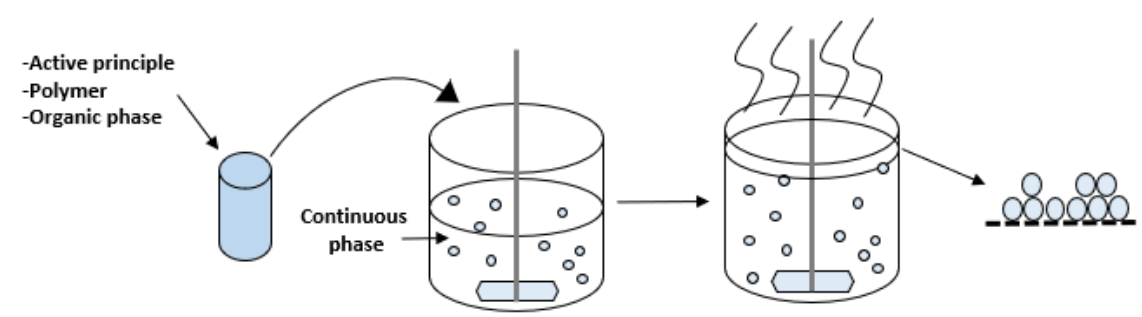

Figure 24. Process of microencapsulation by solvent evaporation.

\subsection{Physical-chemical methods.}

These divide into coacervation/ phase separation and rapid expansion of supercritical fluids. Coacervation and phase separation includes three basic steps: forming three immiscible phases, deposition of the coating, and rigidization of the coating. This consists of three immiscible phases: liquid manufacturing vehicle, core material, and coating material. The core material is dispersed in the coating polymer solution. An immiscible polymer in a liquid state as coating material phase is formed by changing the temperature of polymer solution, adding salt, non-solvent, incompatible polymer to the polymer solution, and inducing polymerpolymer interaction. The second step is the separation of coacervate from the solution. The third step is a deposition of the liquid polymer upon the core material. It is coated by microdroplets of coacervate. In the last step, coalescence of coacervate forms a continuous shell around core particles. The prepared microcapsules are stabilized by cross-linking, desolvation, or heat treatment (Fig. 25) [52].

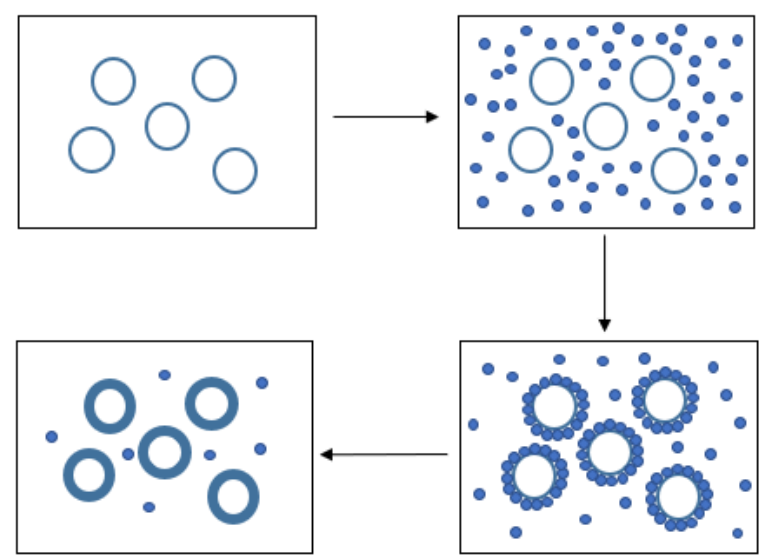

Figure 25. Process of the coacervation. 
In the rapid expansion of supercritical fluids, common supercritical fluids used in polymer encapsulation include supercritical $\mathrm{CO}_{2}$, alkanes ( $\mathrm{C} 2$ to $\left.\mathrm{C} 4\right)$, and nitrous oxide $\left(\mathrm{N}_{2} \mathrm{O}\right)$. Supercritical fluids can largely change the density of supercritical fluids near the critical point via a small change in temperature or pressure. The widest methods used in polymer encapsulation by a rapid expansion of supercritical fluids include rapid expansion of supercritical solution (RESS); gas anti-solvent (GAS); and particles from gas-saturated solution (PGSS).

In rapid expansion of the supercritical solution, the supercritical fluid containing the active ingredient and the shell material is kept under high pressure. A supercritical fluid is then released under atmospheric pressure via a small nozzle. The sudden drop in pressure causes desolvation of the shell material. Finally, the active ingredient (core) is deposited around, forming a coating (Fig. 26).

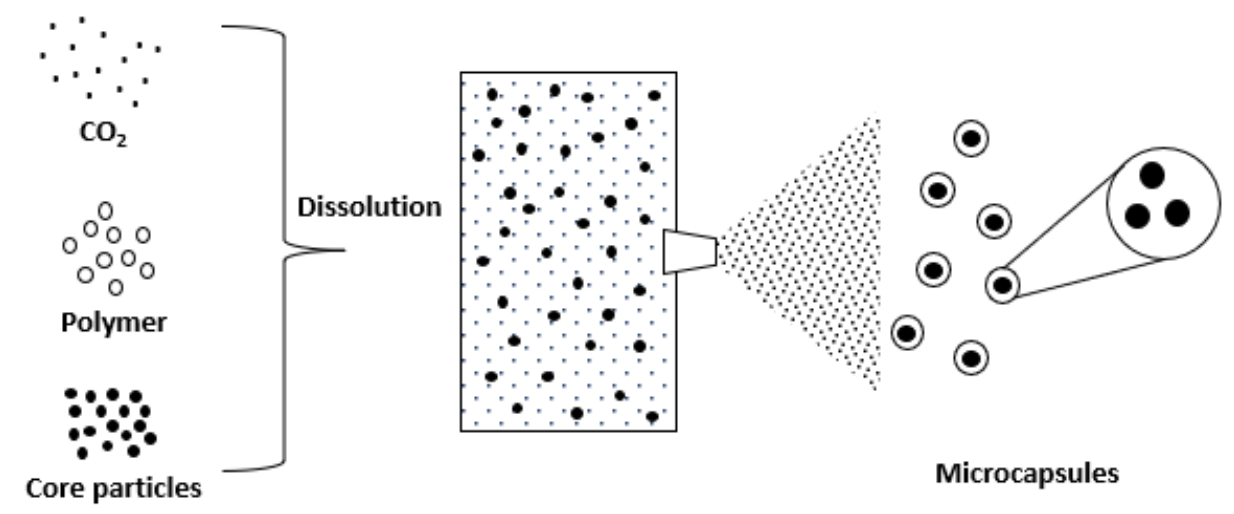

Figure 26. Microencapsulation by the rapid expansion of supercritical solutions.

In the gas anti-solvent $(G A S)$ process, the supercritical fluid is added to the solution of the shell material. Then, the active ingredients are kept under high pressure. This leads to the volume of the solution expanding, resulting in supersaturation and solute precipitation. Therefore, the solute must be soluble in the liquid solvent but not in the solvent and the supercritical fluid mixture. Moreover, the liquid solvent must be miscible with the supercritical fluid (Fig. 27) [54,55].

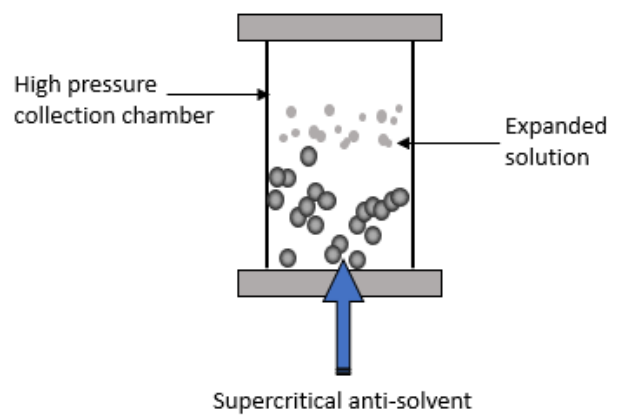

Figure 27. Microencapsulation by gas anti-solvent (GAS) process.

In particles from a gas-saturated solution, supercritical fluid penetrates the shell material, which causes swelling. When the mixture is heated above the glass transition temperature, it makes liquefication of polymer. Upon releasing the pressure, the shell material is allowed to deposit onto the active ingredient. The core and shell materials may not be soluble in the supercritical fluid under this process (Fig. 28) [52,54]. 


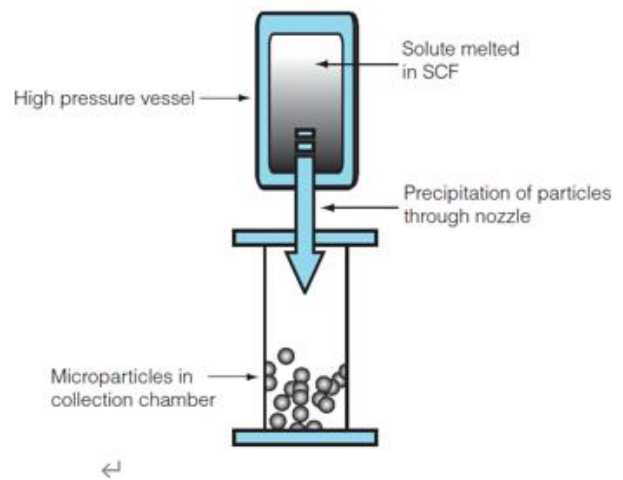

Figure 28. Microencapsulation by particles from a gas-saturated solution.

\section{Conclusion}

Supermolecular devices contain hydrogels and polymers. Gelation and polymerization both are important techniques for synthesizing or manufacturing hydrogels and polymers. Hydrogels (e.g., gelatin and chitosan) are produced via chemical and physical gelation. Polymers (e.g., polyamides) are synthesized through photopolymerization, step-growth, and chain-growth polymerization. Hydrogels and polymers as wall materials entrap core materials via chemical, physical, and physical-chemical microencapsulation.

\section{Funding}

The work described in this paper was fully supported by Hong Kong Metropolitan University Research Grant (R6389, R6401, and PFDS/2021/08).

\section{Acknowledgments}

We would like to express our gratitude to Hong Kong Metropolitan University for the financial support in this project (R6389, R6401, and PFDS/2021/08).

\section{Conflicts of Interest}

The authors declare no conflict of interest.

\section{References}

1. Aida, T. On Supramolecular Polymerization: Interview with Takuzo Aida. Adv Mater 2020, 32, https://doi.org/10.1002/adma.201905445.

2. Yasen, W.; Dong, R.; Aini, A.; Zhu, X. Recent advances in supramolecular block copolymers for biomedical applications. J Mater Chem B 2020, 8, 8219-8231, https://doi.org/10.1039/d0tb01492c.

3. Aida, T.; Meijer, E.W.; Stupp, S.I. Functional supramolecular polymers. Science 2012, 335, 813-817, https://doi.org/10.1126/science.1205962.

4. Aida. T.; Meijer, E.W. Supramolecular Polymers-we've Come Full Circle. Isr. J. Chem 2020, https://doi.org/10.1002/ijch.201900165.

5. Xia, D.; Wang, P.; Ji, X.; Khashab, N.M.; Sessler, J.L.; Huang, F. Functional Supramolecular Polymeric Networks: The Marriage of Covalent Polymers and Macrocycle-Based Host-Guest Interactions. Chem Rev 2020, 120, 6070-6123, https://doi.org/10.1021/acs.chemrev.9b00839.

6. Deligkaris. K.; Tadele, T.; Olthuis, W.; Van den Berg, A. Hydrogel-based devices for biomedical applications. Sens. Actuators B Chem 2010, 147, 765-774, https://doi.org/10.1016/j.snb.2010.03.083.

7. Tang, W.; Chen, C. Hydrogel-Based Colloidal Photonic Crystal Devices for Glucose Sensing. Polymers (Basel) 2020, 12, https://doi.org/10.3390/polym12030625.

8. Chen, Z.; Chen, Y.; Hedenqvist, M.S.; Chen, C.; Cai, C.; Li, H.; Liu, H.; Fu, J. Multifunctional conductive hydrogels and their applications as smart wearable devices. J Mater Chem B 2021, 9, 2561-2583, https://doi.org/10.1039/d0tb02929g. 
9. Imre, B.; Pukánszky, B. Compatibilization in bio-based and biodegradable polymer blends. Eur. Polym. J 2013, 49, 1215-1233, https://doi.org/10.1016/j.eurpolymj.2013.01.019.

10. La Mantia, F.P.; Botta, L.; Mistretta, M.C.; Di, Fiore A.; Titone, V. Recycling of a Biodegradable Polymer Blend. Polymers (Basel) 2020, 12, https://doi.org/10.3390/polym12102297.

11. Ahmed, E.M. Hydrogel: Preparation, characterization, and applications: A review. J Adv Res 2015, 6, 105 121, https://doi.org/10.1016/j.jare.2013.07.006.

12. Odian, G. Principles of Polymerization. Publisher: Wiley 2004.

13. Mukherjee, B.; Chakrabarti, B. Gelation Impairs Phase Separation and Small Molecule Migration in Polymer Mixtures. Polymers (Basel) 2020, 12, https://doi.org/10.3390/polym12071576.

14. Dai, Y.; Zhang, R.; Sun, W.; Wang, T.; Chen, Y.; Tong, Z . Dynamical heterogeneity in the gelation process of a polymer solution with a lower critical solution temperature. Soft Matter 2021, 17, 3222-3233, https://doi.org/10.1039/d0sm02159h.

15. Rubinstein, M.; Colby, R.H. Polymer Physics. Publisher: Oxford University Press 2003.

16. Restrepo-Zapata, N.; Osswald, T.; Hernández-Ortiz, J. Vulcanization of EPDM rubber compounds with and without blowing agents: Identification of reaction events and TTT-diagram using DSC data. Polym. Eng. Sci 2014, 55, 2073-2088, https://doi.org/10.1002/pen.24049.

17. Dörr, D.; Kuhn, U.; Altstädt, V. Rheological Study of Gelation and Crosslinking in Chemical Modified Polyamide 12 Using a Multiwave Technique. Polymers (Basel) 2020, 12, https://doi.org/10.3390/polym12040855.

18. Chen, Y.; Lu, W.; Guo, Y.; Zhu, Y.; Lu, H.; Wu, Y. Superhydrophobic coatings on gelatin-based films: Fabrication, characterization and cytotoxicity studies. RSC Adv 2018, 8, 23712-23719, https://doi.org/10.1039/C8RA04066D.

19. Siangsanoh, C.; Ummartyotin, S.; Sathirakul, K.; Rojanapanthu, P.; Treesuppharat, W. Fabrication and characterization of triple-responsive composite hydrogel for targeted and controlled drug delivery system. $J$. Mol. Liq 2018, 256, 90-99, https://doi.org/10.1016/j.molliq.2018.02.026.

20. Akakuru, O.; Isiuku, B. Chitosan Hydrogels and their Glutaraldehyde-Crosslinked Counterparts as Potential Drug Release and Tissue Engineering Systems - Synthesis, Characterization, Swelling Kinetics and Mechanism. J Phys Chem Biophys 2017, 7, https://doi.org/10.4172/2161-0398.1000256.

21. Capitani, D.; Crescenzi, V.; Segre, A.L. Water in Hydrogels. An NMR Study of Water/Polymer Interactions in Weakly Cross-Linked Chitosan Networks. Macromolecules 2001, 34, 4136-4144, https://doi.org/10.1021/ma002109x.

22. Fan, L.; Ge, X.; Qian, Y.; Wei, M.; Zhang, Z.; Yuan, W.E.; Ouyang, Y. Advances in Synthesis and Applications of Self-Healing Hydrogels. Front Bioeng Biotechnol 2020, 8, https://doi.org/10.3389/fbioe.2020.00654.

23. Xu, Y.; Patino, Gaillez. M.; Rothe, R.; Hauser, S.; Voigt, D.; Pietzsch, J.; Zhang, Y. Conductive Hydrogels with Dynamic Reversible Networks for Biomedical Applications. Adv Healthc Mater 2021, 10, https://doi.org/10.1002/adhm.202100012.

24. D’Agostino, C.; Liuzzi, R.; Gladden, L.; Guido, S. Swelling-induced structural changes and microparticle uptake of gelatin gels probed by NMR and CLSM. Soft Matter, 2017, 13, 2952-2961, https://doi.org/10.1039/C6SM02811J.

25. Buchberger, A.; Saini, H.; Eliato, K.R.; Zare, A.; Merkley, R.; Xu, Y.; Bernal, J.; Ros, R.; Nikkhah, M.; Stephanopoulos, N. Reversible Control of Gelatin Hydrogel Stiffness by Using DNA Cross-linkers. Chembiochem 2021, 22, 1755-1760, https://doi.org/10.1002/cbic.202100030.

26. Mahmood, K.; Sulaiman, S.; Kamilah, H. Review of Fish Gelatin Extraction, Properties and Packaging Applications. Food Science and Quality Management 2016, 56, 47-59.

27. Imani, R.; Rafienia, M.; Hojjati, E.S. Synthesis and characterization of glutaraldehyde-based cross-linked gelatin as a local hemostat sponge in surgery: Anin vitrostudy. Biomed Mater Eng 2013, 23, 211-224, https://doi.org/10.3233/BME-130745.

28. Gafri, H.; Mohamed Zuki, F.; Aroua, M.; Hashim, N. Mechanism of bacterial adhesion on ultrafiltration membrane modified by natural antimicrobial polymers (chitosan) and combination with activated carbon (PAC). Rev Chem Eng 2019, 35, 421-44, https://doi.org/10.1515/revce-2017-0006.

29. Escudero-Oñate, C.; Martínez-Francés, E. A Review of Chitosan-Based Materials for the Removal of Organic Pollution from Water and Bioaugmentation. Publisher: Licensee IntechOpen 2018.

30. Li, Q.; Dunn, E.T.; Grandmaison, W.E.; Goosen, M.F.A. Applications and Properties of Chitosan in Applications of Chitin and Chitosan. Goosen M.F.A.(ed.). Publisher: Technomic Publishing Company, Inc. USA 1997.

31. Cao, J.; Wang, P.; Liu, Y.; Zhu, C.; Fan, D. Double cross-linked HLC-CCS hydrogel tissue engineering scaffold for skin wound healing. Int $J$ Biol Macromol 2020, 155, 625-635, https://doi.org/10.1016/j.ijbiomac.2020.03.236.

32. Marapureddy, S.G.; Hivare, P.; Kumar, S.; Gupta, S.; Thareja, P. Carbamoylated chitosan hydrogels with improved viscoelastic properties and stability for potential 3D cell culture applications. Biomed Mater. 2021, 16, https://doi.org/10.1088/1748-605X/abf88c. 
33. Suganthi, S.; Vignesh, S.; Kalyana Sundar, J.; Vairamuthu R. Fabrication of PVA polymer films with improved antibacterial activity by fine-tuning via organic acids for food packaging applications. Appl Water Sci 2020, 10, https://doi.org/10.1007/s13201-020-1162-y.

34. Tang, X.; Alavi, S. Recent advances in starch, polyvinyl alcohol based polymer blends, nanocomposites and their biodegradability. Carbohydr. Polym 2011, 85, 7-16, https://doi.org/10.1016/j.carbpol.2011.01.030.

35. Baker, M.; Walsh, S.; Schwartz, Z.; Boyan, B. A review of polyvinyl alcohol and its uses in cartilage and orthopedic applications. J. Biomed. Mater. Res 2012, 100B, 1451-1457, https://doi.org/10.1002/jbm.b.32694.

36. Kabir, M.; Kim, H.; Lee, C.; Choi, S. Highly Proton Conductive Poly(vinyl acetate)/Nafion® Composite Membrane for Proton Exchange Membrane Fuel Cell Application. J. Nanosci. Nanotechnol 2018, 18, 65366540, https://doi.org/10.1166/jnn.2018.15668.

37. Gomaa, M.; Hugenschmidt, C.; Dickmann, M.; Abdel-Hady, E.; Mohamed, H.; Abdel-Hamed, M. Crosslinked PVA/SSA proton exchange membranes: Correlation between physiochemical properties and free volume determined by positron annihilation spectroscopy. Phys. Chem. Chem. Phys 2018, 20, 28287-28299, https://doi.org/10.1039/C8CP05301D.

38. Imre, B.; Pukánszky, B. Compatibilization in bio-based and biodegradable polymer blends. Eur. Polym. J 2013, 49, 1215-1233, https://doi.org/10.1016/j.eurpolymj.2013.01.019.

39. Han, F.; Zhu, C.; Chen, L.; Wicks, J.; Li, B. Bio-Instructive Scaffolds For Bone Regeneration. Bio-Instructive Scaffolds for Musculoskeletal Tissue Engineering and Regenerative Medicine 2017, 55-84, https://doi.org/10.1016/B978-0-12-803394-4.00003-3.

40. Mai, D.; Schroeder, C. Single polymer dynamics of topologically complex DNA. Curr Opin Colloid Interface Sci 2016, 26, 28-40, https://doi.org/10.1016/j.cocis.2016.08.003.

41. White, J.; De, S. Rubber Technologist's Handbook. Publisher: Shrewsbury: ISmithers Rapra Pub, 2001.

42. Nic, M.; Jirat, J.; Kosata, B. Compendium of Chemical Terminology. Publisher: Durham, NC International Union of Pure and Applied Chemistry 2012.

43. Young, R.; Lovell, P. Introduction to Polymers. Boca Ratón. Publisher: FL CRC Press 2011.

44. Brown, W.; Anslyn, E.; Foote, C.; Iverson, B. Organic Chemistry. Publisher: Cengage 2018.

45. Bossion, A.; Heifferon, K.; Meabe, L.; Zivic, N.; Taton, D.; Hedrick, J.; Long, T.; Sardon, H. Opportunities for organocatalysis in polymer synthesis via step-growth methods. Prog. Polym. Sci 2019, 90, 164-210, https://doi.org/10.1016/j.progpolymsci.2018.11.003.

46. Zakaria, Z.; Izzah, Z.; Jawaid, M.; Hassan, A. Effect of degree of deacetylation of chitosan on thermal stability and compatibility of chitosan-polyamide blend. Bioresources 2012, 7, 5568-5580, https://doi.org/10.15376/biores.7.4.5568-5580.

47. Palmer, R. Polyamides, Plastics. Encyclopedia of Polymer Science and Technology 2011, https://doi.org/10.1002/0471440264.pst251.

48. Deopura, B.; Alagirusamy, R.; Jushi, M.; Gupta, B. Polyesters and polyamides. Publisher: Woodhead 2008.

49. Rosa, N.; Martins, G.; Bastos, M.; Góis, J.; Coelho, J.; Marques, J.; Magalhães, F. Preparation of robust polyamide microcapsules by interfacial polycondensation of p-phenylenediamine and sebacoyl chloride and plasticization with oleic acid. J. Microencapsul 2015, 32, 349-357, https://doi.org/10.3109/02652048.2015.1028494.

50. Munin, A.; Edwards-Lévy, F. Encapsulation of Natural Polyphenolic Compounds; a Review. Pharmaceutics 2011, 3, 793-829, https://doi.org/10.3390/pharmaceutics3040793.

51. Zhang, H.; Li, W.; Huang, R.; Wang, N.; Wang, J.; Zhang, X. Microstructure regulation of microencapsulated bio-based n-dodecanol as phase change materials via in situ polymerization. New J. Chem 2017, 41, 1469614707, https://doi.org/10.1039/C7NJ02864D.

52. Jyothi, N.; Prasanna, P.; Sakarkar, S.; Prabha, K.; Ramaiah, P.; Srawan, G.Y. Microencapsulation techniques, factors influencing encapsulation efficiency. J. Microencapsul 2010, 27, 187-197, https://doi.org/10.3109/02652040903131301.

53. Li, M.; Rouaud, O.; Poncelet, D. Microencapsulation by solvent evaporation: State of the art for process engineering approaches. Int. J. Pharm 2008, 363, 26-39, https://doi.org/10.1016/j.ijpharm.2008.07.018.

54. Ginty, P.; Whitaker, M.; Shakesheff, K.; Howdle, S. Drug delivery goes supercritical. Mater. Today Commun 2005, 8, 42-48, https://doi.org/10.1016/S1369-7021(05)71036-1.

55. Liu, G.; Li, J.; Deng, S. Applications of Supercritical Anti-Solvent Process in Preparation of Solid Multicomponent Systems. Pharmaceutics 2021, 13, https://doi.org/10.3390/pharmaceutics13040475. 